Pesq. Vet. Bras. 36(4):317-321, abril 2016 DOI: $10.1590 / \mathrm{S} 0100-736 \mathrm{X} 2016000400011$

\title{
Descrição morfológica de duas espécies de Sapajus encontradas na Paraíba: S. libidinosus e o recém- redescoberto e já criticamente ameaçado $S$. flavius ${ }^{1}$
}

\author{
Max B.M. Bacalhao ${ }^{2}$, Millena O. Firmino², Raul A.S. Siqueira ${ }^{2}$, Ayodhya C. Ramalho ${ }^{2}$, \\ Tarsila A. Cavalcante ${ }^{3}$, Thiago F.L. Nery ${ }^{4}$ e Ricardo R. Guerra ${ }^{2 *}$
}

\begin{abstract}
Bacalhao M.B.M., Firmino M.O., Siqueira R.A.S., Ramalho A.C., Cavalcante T.A., Nery T.F.L. \& Guerra R.R. 2016. [Morphological description of two species of Sapajus found in Paraíba: $S$. libidinosus and the newly discovered and endangered $\boldsymbol{S}$. flavius.] Descrição morfológica de duas espécies de Sapajus encontradas na Paraíba: S. libidinosus e o recém-redescoberto e já criticamente ameaçado S. flavius. Pesquisa Veterinária Brasileira 36(4):317-321. Programa de Pós-Graduação em Ciência Animal, Centro de Ciências Agrárias, Universidade Federal da Paraíba, Campus II, Areia, PB 58397-000, Brazil. E-mail: rromaoguerra@gmail.com

Sapajus flavius and S. libidinosus are two species of capuchin monkeys found in the state of Paraíba, Brazil. S. flavius or Macgrave's capuchin monkey was recently rediscovered and is now critically endangered, found only in remaining fragments of Atlantic forest in the coastal region of the Brazian Northeast. S. libidinosus has a larger population and distribution, coexisting with S. flavius in Paraíba, however living in the western part of the state, in the semi-arid. Since there is a lack of knowledge about these species, a morphologic description of them was made, involving external and gastrointestinal tract biometry, visceral topography and anatomic description. Although the literature describes $S$. flavius as the smaller monkey of the Sapajus genus, we conclude that there are no morphological differences between these species. Such information contributes to best management practices for conservation of the species and to the correct taxonomic classification of the genus recently modified.
\end{abstract}

INDEX TERMS: Conservation, taxonomy, morphology, Sapajus libidinosus, Sapajus flavius, Macgrave's capuchin monkey.

RESUMO.- Sapajus flavius e S. libidinosus são duas espécies de macacos-prego encontrados no Estado da Paraíba. $S$. flavius ou macaco-prego-galego foi recentemente redescoberto e está criticamente ameaçado de extinção, sendo encontrado em fragmentos remanescentes de mata Atlântica do litoral do RN, PB, PE e AL. S. libidinosus tem uma distribuição e população maior, coexistindo de $S$. flavius na PB, entretanto, ocupando o oeste da PB, na caatinga. Frente à lacuna no conhecimento sobre estas espécies foi realizada

\footnotetext{
${ }^{1}$ Recebido em 8 de junho de 2015.

Aceito para publicação em 17 de janeiro de 2016.

${ }^{2}$ Programa de Pós-Graduação em Ciência Animal, Centro de Ciências Agrárias, Universidade Federal da Paraíba (UFPB), Campus II, Areia, PB 58397000, Brasil. *Autor para correspondência: rromaoguerra@gmail.com

${ }^{3}$ Centro de Triagem de Animais Selvagens, ICMBio/IBAMA, Mata do Amém, Cabedelo, PB 58310-000, Brasil.

${ }^{4}$ Zoo Arruda Câmara, Av. Gouveia Nóbrega, Roger, João Pessoa, PB 58020-325, Brasil.
}

a descrição morfológica das mesmas, envolvendo biometria externa, dos órgãos do trato gastrointestinal, topografia visceral e a descrição anatômica. Apesar da literatura descrever S. flavius como o menor dos Sapajus, concluímos que não há diferenças morfológicas entre as espécies estudadas. Tais informações servem de subsídios para melhores manejos visando a conservação das espécies e ajudando na classificação taxonômica desse gênero recentemente modificado.

TERMOS DE INDEXAÇÃO: Conservação, taxonomia, morfologia, Sapajus libidinosus, Sapujus flavius, macaco-prego-galego.

\section{INTRODUÇ̃̃o}

O macaco-prego-dourado ou macaco-prego-galego (Sapajus flavius) é uma espécie de macaco-prego que há muito se acreditava estar extinta, sendo redescoberta recentemente (Pontes et al. 2006). Tais autores por pensar que 
se tratava de uma nova espécie, a denominou como Cebus queirozi (Oliveira \& Langguth 2006, Valença-Montenegro 2011). Atualmente existem apenas 26 populações ou áreas de relato com um número não catalogado de indivíduos em fragmentos de Mata Atlântica do Rio Grande do Norte, Paraíba e Pernambuco (Oliveira \& Langguth 2006, Guimarães 2012). A espécie está incluída na Lista Vermelha da International Union of Conservation of Natura como criticamente ameaçada de extinção (INCN 2015).

Até recentemente (Lynch Alfaro et al. 2012a, 2012b, Garber et al. 2012) S. flavius era chamado de Cebus flavius, ainda hoje tal espécie é chamada de $C$. flavius por alguns autores (Valença-Montenegro 2011, Teixeira et al. 2013). Tal mudança na classificação taxonômica ocorreu primeiramente com Silva (2001), que propôs a divisão dos macacos-pregos em dois subgêneros: Cebus para os caiararas, mais esguios, distribuídos da Amazônia para o norte, e Sapajus para os macacos-pregos, mais robustos e caracterizados por um topete na cabeça, espalhados da Amazônia para o sul, na mata atlântica, cerrado e caatinga (Valença-Montenegro 2011, Lynch Alfaro et al. 2012a). Hoje, através de amplas análises genéticas (Lynch Alfaro et al. 2012b, Russell et al. 2013), ficou demonstrado que os Cebus e Sapajus são gêneros distintos. Sendo assim, o C. flavius passou a ser classificado como $S$. flavius, espécie a pouco conhecida apenas por uma pintura do século XVIII, com quase nenhum estudo científico (Guimarães 2012, Teixeira et al. 2013) e já criticamente ameaçado de extinção.

No Estado da Paraíba outra espécie de Sapajus coexiste, entretanto, diferentemente da primeira que habita principalmente a faixa litorânea e fragmentos de mata atlântica do agreste, o Sapajus libidinosus habita principalmente a caatinga (Lynch Alfaro et al. 2012b, Rylands et al. 2013). S. libidinosus ocorre no Brasil central e nordeste, a oeste e norte do rio São Francisco, e leste do rio Araguaia, ocorrendo nos estados do Maranhão, Piauí, leste e centro do Rio Grande do Norte, noroeste da Paraíba e oeste de Pernambuco e Alagoas. Há ocorrência também em Minas Gerais, ao norte do rio grande (Rylands et al. 2013). Seu estado de conservação, ao contrário do S. flavius, é pouco preocupante (Rylands \& Kierulff 2008, Rylands et al. 2013).

A caracterização biométrica para essas duas espécies de primatas neotropicais são incipientes. Existe apenas um trabalho publicado abordando a biometria externa de um espécime de $S$. flavius (Pontes et al. 2006) e nenhum para S. libidinosus. Estudos sobre a morfologia geral de tais espécies inesistem. Mesmo para outras espécies de primatas não-humanos neotropicais são escassos os estudos, sendo a maioria dos estudos envolvendo primatas do "velho mundo".

Sendo assim, frente às lacunas de conhecimento, o presente trabalho objetivou caracterizar morfologicamente duas dessas espécies de primatas neotropicais encontradas no Estado da Paraíba, o S. flavius e o S. libidinosus, através de populações mantidas em cativeiro, contribuindo para a elucidação da divisão taxonômica da subfamília em questão, ainda controversa por alguns autores (Guimarães 2012). Além de prover subsídios para melhores manejos clínicos, cirúrgicos e nutricionais, bem como, gerar conhe- cimento para auxiliar técnicas de diagnóstico por imagens envolvendo estas e outras espécies de primatas.

\section{MATERIAL E MÉTODOS}

Os macacos-pregos Sapujus flavius estudados foram oriundos de uma população de 8 indivíduos vivos (7 machos e 1 fêmea), mantidos em cativeiro no CETAS/IBAMA em Cabedelo, PB, situado na Mata do Amém; 4 carcaças (2 machos e 2 fêmea), oriundos também de animais que vieram a óbito no CETAS/IBAMA e 1 fêmea oriunda do Parque Zoobotânico Arruda Câmara em João Pessoa, PB (doado pelo CETAS). Os S. libidinosus estudados foram oriundos de animais que morreram no CETAS/IBAMA, sendo 7 machos e 5 fêmeas. Todos os animais eram adultos ou jovens adultos, e oriundos de apreensões realizadas pelo IBAMA no Estado da Paraíba. A execução do trabalho se deu através do convênio com o IBAMA no. 02019.00129/2009-12 e liberação da Comissão de Ética no Uso de Animais (CEUA) da Universidade Federal da Paraíba no. 0710/12 e SISBIO no. 35565-1.

Os animais vivos foram contidos fisicamente com Puçá, em seguida, contidos quimicamente com Xilazina $2 \%(2 \mathrm{mg} / \mathrm{kg})$ associado a Cetamina $10 \%(10 \mathrm{mg} / \mathrm{kg})$. Os pesos dos animais para preparação das doses foram subjetivos, avaliando a relação tamanho/massa corporal. Após a sedação os mesmos foram pesados em balança pediátrica eletrônica (Welmy) e as medidas biométricas externas aferidas, sendo elas: cabeça-corpo, cauda, patas, orelha, tíbia, fêmur, úmero e rádio-ulna, circunferência do crânio, tórax e abdômen (Pontes et al. 2006).

As carcaças também tiveram o peso e a biometria aferida. As mesmas foram submetidos a necropsia sendo realizada uma incisão na linha Alba. 0 dente canino a as vísceras do trato gastrointestinal (TGI) (língua, esôfago cervical e torácico, curvatura maior do estômago, intestino e ceco) foram medidos com auxílio de fita métrica e paquímetro digital. Também foi avaliada a relação intestino/tamanho corpóreo. A topografia das vísceras e a morfologia dos órgãos do sistema digestório e respiratório foram descritas e catalogadas através de câmera digital (Kodak EasyShare M340, 10.2 MP). Os parâmetros biométricos foram comparados entre as espécies pelo Test t $(P \leq 0,05)$ no programa MiniTab.

Foi realizada maceração e clareamento do crânio dos espécimes estudados para acervo didático e comparação de formato e arcada dentária.

\section{RESULTADOS E DISCUSSÃO}

\section{Características fenotípicas e biométricas}

As espécies estudadas possuíam fenótipos diferentes. As características fenotípicas de Sapajus flavius são descritas como diferentes das outras espécies de Sapajus sp., devido seu menor peso, topete menos evidenciado e por possuírem os pelos do corpo uniformemente amarelo-dourado (Pontes et al. 2006, Oliveira \& Langguth 2006). Os animais estudados corroboram essas descrições, a exceção do peso. Verificamos que $S$. flavius possuiu o mesmo peso de $S$. libidinosus, contrariando a literatura, pelo menos nesses espécimes do Estado da Paraíba, que foram apreendidas pelo CETAS/IBAMA.

Os $S$. flavius estudados apresentaram dimorfismo sexual, uma vez que os machos adultos eram mais robustos e tinham uma prega escura sem pelos na região ventral do pescoço, característica essa descrita apenas no espécime de Pontes et al. (2006). Tal dimorfismo sexual é menos notório em S. libidinosus e nas outras espécies de Sapajus (Rylands et al. 2013). 
S. libidinosus possuia coloração que tende a um amarelo ou bege, com os membros de cor escura tendendo ao preto, assim como o topete, que possuia forma espessa, conforme já descrito para a espécie em outras regiões do Brasil (Rylands et al. 2005). Tal espécie já fora classificada como subespécie de $S$. apella (Groves et al. 2005). Os S. apella são mais escuros, mais numerosos, apresentam maior distribuição geográfica entre os Sapajus, possuem diversas subespécies e extendem-se até outros países como Equador, Bolívia e Peru (Alves et al. 2012, Eisenberg \& Redford 1999, Groves et al. 2005, Lynch Alfaro et al. 2012a).

As espécies de Sapajus estudadas, assim como as demais, diferem dos Cebus, anteriormente mantidos no mesmo gênero (Silva et al. 2001) por serem mais robustos (Lynch Alfaro et al. 2012b) e pela maior proeminência cranial (Silva et al. 2001). As medidas biométricas externas de S. flavius e S. libidinosus encontram-se no Quadro 1.

Foi verificado que não há diferenças biométricas externas significativas entre $S$. flavius e $S$. libidinosus, entretanto, quando esses foram comparados com 3 exemplares de $S$. apella (dados não publicados), também mantidos no CETAS/IBAMA, observou que estes tendem a possuir peso, comprimento de fêmur, tíbia, cauda, circunferência crânio e de abdômen maiores que as outras duas espécies. Tais resultados não corroboram Pontes et al. (2006); os mesmos descrevem S. flavius, no Estado de Pernambuco como sendo menor que as demais espécies de Sapajus, entretanto, demonstramos que pelo menos no Estado da Paraíba, $S$. flavius e $S$. libidinosus também são semelhantes biometricamente, inclusive com crânios semelhantes.

Valença-Montenegro (2011), estudando populações livres de $S$. flavius, também no Estado da Paraíba, descreve machos e fêmeas (jovens e adultos) $(2,172 \pm 320,95 \mathrm{~kg}$ e $1,657 \pm 231,55 \mathrm{~kg}$, respectivamente), com pesos semelhantes aos encontrados nesse trabalho. Ambos os valores ainda são menores que o macho de $S$. apella $(2,505 \mathrm{~kg})$ mantido no mesmo CETAS/IBAMA de Cabedelo, PB. Pontes et al. (2006) descrevem, em uma população de Pernambuco, um S. flavius ainda mais pesado, com $2,920 \mathrm{~kg}$ e medidas para cabeça-corpo, pé, tíbia, fêmur, mão, úmero e rádio, respectivamente de $40,12,15,3,12,8,4,11,6$ e 12,6cm. Entretanto, esse animal possuiu cauda $(41 \mathrm{~cm})$ e orelha $(2,9 \mathrm{~cm})$ menor que as dos espécimes do presente estudo. A descrição biométrica externa do espécime de Pontes et al. (2006) é a única existente para $S$. flavius até o presente trabalho.

Apesar de mais numerosa e conhecida que $S$. flavius, não existem estudos biométricos para o $S$. libidinosus, apenas estudos comportamentais, visto que ambas as espécies utilizam-se de ferramentas rudimentares, fato raro, mesmo para primatas (Canale et al. 2009, Falótico et al. 2011).

Mesmo para outros primatas neotropicais, valores biométricos são raros. Para circunferência abdominal os únicos valores encontrados são para bugio-ruivo (machos 35,5; fêmeas $24,9 \mathrm{~cm}$ ), macaco-da-noite (macho 8,6; fêmea $7,0 \mathrm{~cm}$ ) e mico-de-cheiro (macho 5,56; fêmea $3,07 \mathrm{~cm}$ ) (Souza 2013). Desses, apenas os bugios-ruivos apresentam tal medida maior que nas espécies do presente estudo.

A biometria do TGI (Quadro 1) também demonstrou não haver diferenças entre as espécies estudadas. Tais se-
Quadro 1. Medidas biométricas externas, peso e medidas biométricas internas de macacos das espécies Sapajus flavius e Sapajus libidinosus oriundas do CETAS-IBAMA, Cabedelo/PB e do Zoológico Arruda Câmara, João Pessoa/PB

\begin{tabular}{lcc}
\hline $\begin{array}{l}\text { Medidas biométricas } \\
\text { externas }\end{array}$ & S. flavius $(\mathrm{n}=13)$ & S. libidinosus $(\mathrm{n}=12)$ \\
\hline & \multicolumn{2}{c}{$\mathrm{cm}$} \\
Cabeça-Corpo & $38,400 \pm 4,615^{\mathrm{a}}$ & $36,292 \pm 3,093^{\mathrm{a}}$ \\
Cauda & $43,000 \pm 4,528^{\mathrm{a}}$ & $42,083 \pm 2,811^{\mathrm{a}}$ \\
Pé & $7,660 \pm 0,365^{\mathrm{a}}$ & $7,800 \pm 0,886^{\mathrm{a}}$ \\
Pé com dedo & $11,300 \pm 0,731^{\mathrm{a}}$ & $11,800 \pm 0,789^{\mathrm{a}}$ \\
Tíbia & $12,600 \pm 0,894^{\mathrm{a}}$ & $12,542 \pm 1,322^{\mathrm{a}}$ \\
Fêmur & $11,900 \pm 1,025^{\mathrm{a}}$ & $11,708 \pm 0,689^{\mathrm{a}}$ \\
Mãos & $4,320 \pm 0,356^{\mathrm{a}}$ & $4,050 \pm 0,358^{\mathrm{a}}$ \\
Mãos com dedo & $7,480 \pm 0,415^{\mathrm{a}}$ & $7,725 \pm 0,685^{\mathrm{a}}$ \\
Úmero & $10,800 \pm 1,037^{\mathrm{a}}$ & $10,042 \pm 1,233^{\mathrm{a}}$ \\
Rádio & $11,000 \pm 0,707^{\mathrm{a}}$ & $10,333 \pm 0,913^{\mathrm{a}}$ \\
Circ. Crânio & $23,400 \pm 1,517^{\mathrm{a}}$ & $22,833 \pm 1,193^{\mathrm{a}}$ \\
Circ. Tórax & $24,700 \pm 2,168^{\mathrm{a}}$ & $22,933 \pm 1,621^{\mathrm{a}}$ \\
Circ. Abdomen & $17,520 \pm 2,297^{\mathrm{a}}$ & $16,875 \pm 2,144^{\mathrm{a}}$ \\
Orelha & $3,700 \pm 0,742^{\mathrm{a}}$ & $3,645 \pm 0,450^{\mathrm{a}}$ \\
& & \\
Peso & $1,746 \pm 0,374 \mathrm{a}$ & $1,565 \pm 0,303 \mathrm{a}$ \\
& Macho Fêmea & Macho Fêmea \\
& $1,90 \pm 0,621,67 \pm 0,29$ & $1,65 \pm 0,251,45 \pm 0,36$ \\
Medidas biométricas & S. libidinosus $(\mathrm{n}=5)$ & S. flavius $(\mathrm{n}=12)$
\end{tabular}
do TGI

\begin{tabular}{lcr} 
& \multicolumn{2}{c}{$\mathrm{cm}$} \\
Dente Canino & $1,301 \pm 0,283^{\mathrm{a}}$ & $1,351 \pm 0,037^{\mathrm{a}}$ \\
Língua & $5,025 \pm 0,171^{\mathrm{a}}$ & $5,175 \pm 0,386^{\mathrm{a}}$ \\
Esôfago Cervical & $5,581 \pm 2,323^{\mathrm{a}}$ & $5,325 \pm 1,053^{\mathrm{a}}$ \\
Esôfago Torácico & $7,033 \pm 1,95^{\mathrm{a}}$ & $6,050 \pm 1,684^{\mathrm{a}}$ \\
Estômago Curvatura maior & $17,089 \pm 2,080^{\mathrm{a}}$ & $19,001 \pm 2,464^{\mathrm{a}}$ \\
Intestino & $220,203 \pm 11,967^{\mathrm{a}}$ & $218,821 \pm 6,702^{\mathrm{a}}$ \\
Ceco & $4,461 \pm 1,140^{\mathrm{a}}$ & $5,125 \pm 0,629^{\mathrm{a}}$ \\
Relação intestino/ & $5,963 \pm 0,702^{\mathrm{a}}$ & $5,892 \pm 0,646^{\mathrm{a}}$
\end{tabular}

tamanho corporal

Comparação das médias das variáveis de biometria externa, interna e peso aplicando-se o Test $t$. Letras diferentes representam significância ao nível de $5 \%$.

melhanças demonstram a paridade entre os hábitos alimentares das espécies, apesar dos habitats distintos. Não há na literatura estudos acerca da biometria do TGI de primatas neotropicais, sendo esta a primeira descrição.

\section{Topografia}

Observou-se consonância visceral entre as duas espécies estudadas. Seguindo sentido crânio-caudal, observou a língua tendo como limite rostral os dentes incisivos, caudal a laringe, lateral os dentes pré-molares e molares, dorsal o palato duro e ventral com o músculo milo-hióide. 0 esôfago estendeu-se desde a laringe cranialmente até o estômago caudalmente, percorrendo ao lado da taqueia na vista esquerda do pescoço, passando pela cavidade torácica e penetrando na cavidade abdominal com limite no cárdia. A traqueia seguiu medialmente no pescoço vista ventral, estendendo-se da laringe até a bifurcação dos dois brônquios na cavidade torácica. 0 pulmão foi dividido em dois hemipulmões independentes. 0 fígado encontrou-se em íntimo contato com o diafragma pela face diafragmática e com o estômago, curvatura menor, pela face visceral, sendo dividido em lobações, assim como descrito para S. apella (Alves et al. 2007). 0 estômago estendeu-se do esôfago cranial- 

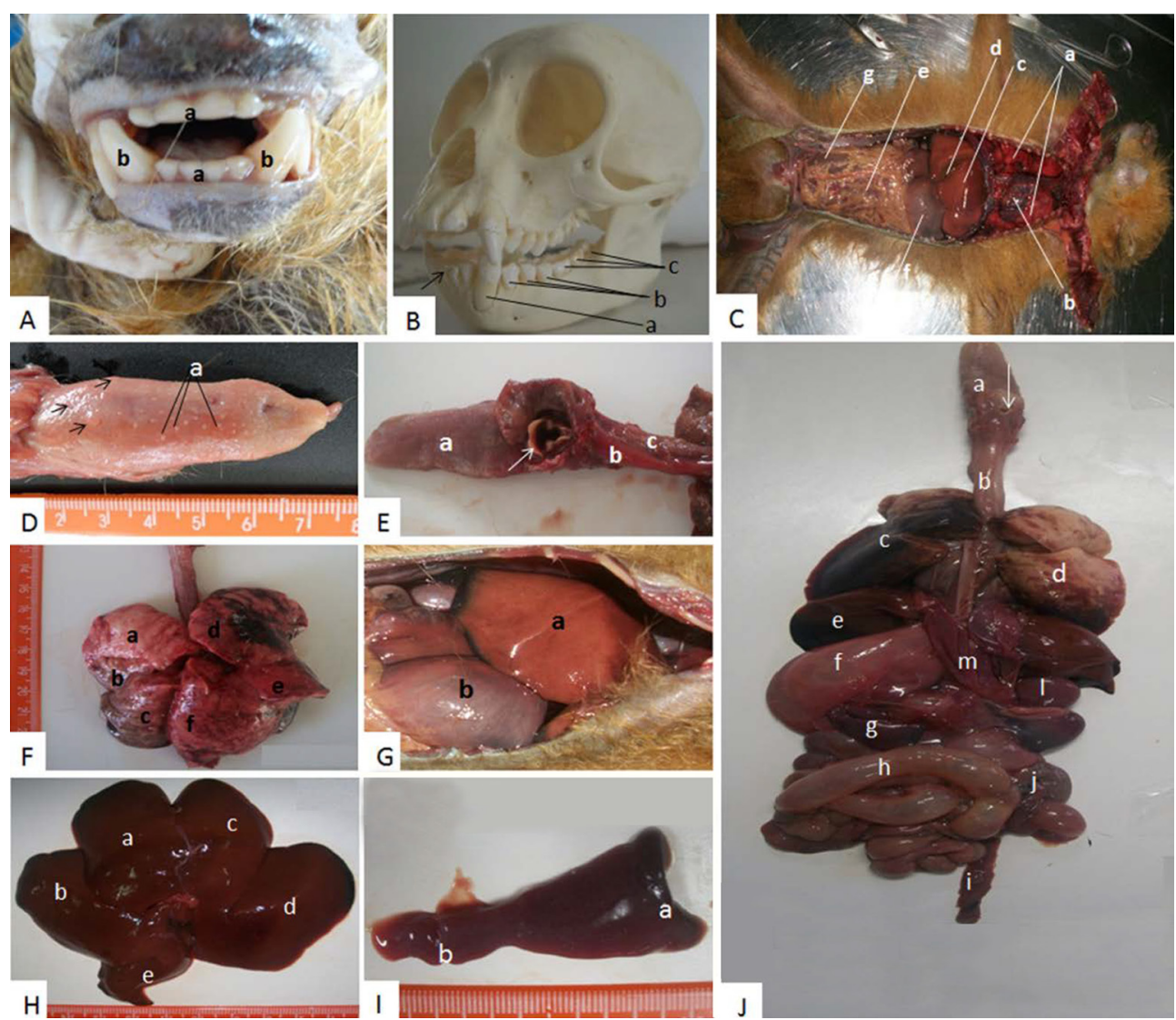

Fig.1. Imagens fotográficas representativas de Sapajus flavius e Sapajus libidinosus. (A) Arcada dentária: a-incisivos superiores e inferiores, b-caninos inferiores. (B) Crânio macerado de S. flavius: a-canino inferior, b-pré-molares inferiores, c-molares inferiores. (C) Topografia in situ de S. flavius: a-pulmões, b-coração, c-fígado, d-vesícula biliar, e-omento, f-estômago, g-intestino grosso. (D) Língua: a-papilas fungiformes, setas-papilas valadas. (E) Língua: a-língua, b-esôfago, c-traqueia, seta-epiglote. (F) Pulmão: a-lobo cranial direito, b-lobo medial direito, c-lobo caudal direito, d- porção cranial do lobo cranial esquerdo, e-porção caudal do lobo cranial esquerdo, f-lobo caudal esquerdo. (G) Cavidade abdominal: a-fígado, b-estômago. (H) Fígado: a-lobo hepático medial direito, b-lobo hepático lateral direito, c-lobo hepático medial esquerdo, d-lobo hepático lateral esquerdo, e-processo caudado do lobo caudado. (I) Baço: a-extremidade ventral, b-extremidade dorsal. (J) Imagem topográfica ex situ das vísceras de S. flavius: a-língua, b-esôfago, c-pulmão direito, d-pulmão esquerdo, e-fígado, f-estômago, g-baço, h-intestino grosso, i-cólon descendente, j-intestino delgado, l-rim, m-diafragma, seta-entrada da traqueia.

mente até o intestino delgado caudalmente; sendo localizado na porção crânio-lateral esquerda do abdomen. As alças intestinais se encontraram em contato com o estômago na porção lateral-esquerda, com o fígado crânio-lateral direito e com o baço na porção ventral (Fig.1C, J).

\section{Anatomia}

Anatomicamente os órgãos também apresentaram semelhanças entre as duas espécies, sendo assim, descritos conjuntamente. As espécies apresentaram quantidade total de 36 dentes, distribuídos em duas arcadas dentárias, superior e inferior, com fórmula dentária I2, C1, PM3, M3 em cada, assim como todos os gêneros de Platyrrhiri, a exceção de Callitrichinae (Pinto 2008, Pais 2011) (Fig.1A,B). Na língua foi possível visualizar macroscopicamente 3 papilas valadas localizadas na base da língua, sendo duas paralelas entre si e uma numa posição mais centro-caudal. As papilas filiformes puderam ser observadas por toda extensão da língua (Fig.1D) corroborando Lobo et al. (2014) para catita. A traqueia estava situada caudal a laringe (Fig.1E), seguindo medialmente até sua entrada na cavidade torácica e con- tinua até sua bifurcação terminal sobre o coração. A porção torácica desvia-se para a direita, onde cruza o arco aórtico e relaciona-se latero-dorsalmente com o esôfago. Os anéis de cartilagem possuem formato de "C" com músculo liso completando a circunferência, á semelhança de carnívoros, equídeos (König \& Liebich 2004), S. apella (Leonel et al. 2014) e macaco-de-cheiro (Pinheiro et al. 2012).

Os hemipumões foram divididos em lobações, o lado direito possuiu lobos cranial, médio, caudal e acessório; o lado esquerdo possuiu parte cranial e caudal do lodo cranial e lobo caudal, a semelhança de lêmures (Makungu et al. 2014) e sauim (Chagas et al. 2012). 0 hemipulmão direito era maior; ambos apresentaram uma base (superfície diafragmática), um ápice, duas extremidades (costal e medial) e três bordas (dorsal, ventral e basal) (Fig.1F) como a maioria das espécies (König \& Liebich 2004).

0 estômago estava localizado na porção crânio-lateral esquerda do abdômen (Figura 1G) apresentando formato comum à carnívoros domésticos (Dyce et al. 2010). 0 fígado encontrava-se em íntimo contato com o diafragma pela face diafragmática, corroborando Alves et al. (2007) para $S$. 
apella e com o estômago (curvatura menor), pela face visceral. Sendo as lobações: lobo lateral esquerdo, lobo medial esquerdo, lobo quadrado, lobo medial direito, lobo lateral direito e processo caudado (Fig.1H), sendo essa lobação comparável entre os animais domésticos, ao suíno (König \& Liebich 2004). 0 baço estava localizado na região hipogástrica esquerda e apresentava coloração vermelho-vivo, possuindo extremidade dorsal, mais afilada, e extremidade ventral, mais larga (Fig.1I), dentre os animais domésticos, o que mais se aproxima em formato é o do cão (Dyce et al. 2010). Tais informações anatômicas para essas duas espécies têm importância, por servirem como subsídios para intervenções clínicas e cirúrgicas nessas espécies, podendo tais descrições serem extrapoladas para outras espécies de primatas.

\section{CONCLUSÕES}

0 presente estudo estabelece pela primeira vez o perfil morfológico de Sapajus flavius e de S. libidinosus encontrado na Paraíba.

Ao contrário do que é encontrado na literatura, que $S$. flavius não é menor e mais leve que as demais espécies do gênero Sapajus, sendo não significantes as diferenças para Sapajus libidinosus.

Tais informações servem de subsídios para técnicas de diagnóstico por imagem, para melhores manejos nutricional, clínico e cirúrgico das espécies estudadas, visando sua conservação, além de ajudar na classificação taxonômica desse gênero recentemente modificado.

\section{REFERÊNCIAS}

Alves F.R., Costa F.B., Arouche M.M.S., Barros A.C.E., Miglino M.A., Vulcano L.C. \& Guerra P.C. 2007. Avaliação ultra-sonográfica do sistema urinário, fígado e útero do macaco-prego, Cebus apella. Pesq. Vet. Bras. 27:377382.

Alves F.R., Costa F.B., Machado P.P., Diniz A.N., Araújo A.V.C., Ambrósio C.E. \& Guerra P.C. 2012. Anatomical and radiographic appearance of the capuchin monkey thoracic cavity (Cebus apella). Pesq. Vet. Bras. 32:13451350.

Canale G.R., Guidorizzi C.E., Kierulff M.C. \& Gatto C.A. 2009. First record of tool use by wild populations of the yellow-breasted capuchin monkey (Cebus xanthosternos) and de new records for the bearded capuchin (Cebus libidinosus). Am. J. Primatol. 71:366-372.

Chagas K., Pinheiro V., Lima A.R., Corrêa L., Branco E. \& Albuquerque A.C.A. 2012. Estudo anatômico da traqueia e pulmões do saguim Saguinus midas (Linnaeus, 1758). Arch. Vet. Sci. 17:527-529.

Dyce K.M., Sack W.O. \& Wensing J.G. 2010. Tratado de Anatomia Veterinária. Guanabara Koogan, Rio de Janeiro. 543p.

Eisenberg J.F. \& Redford K.H. 1999. Mammals of the Neotropics. The Central Neotropics Ecuador, Peru, Bolivia, Brazil. The University of Chicago Press, Chicago. 609p.

Falótico T. 2011. Uso de ferramentas por macacos-pregos (Sapajus libidinosus) do parque nacional Serra da Capivara, PI. Tese de Doutorado em Psicologia, Instituto de Psicologia, Universidade de São Paulo, São Paulo, SP. 170p.

Garber P.A., Gomes D.F. \& Bicca-Marques J.C. 2012. Experimental field study of problem-solving using tools in free-ranging capuchins (Sapajus nigritus, formerly Cebus nigritus). Am. J. Primatol. 74:344-358.

Groves C. 2005. Order primates, p.136-138. In: Wilson D.E. \& Reeder D.M. (Eds), Mammal Species of the World. Johns Hopkins University, Baltimore.

Guimarães M. 2012. Ramificações Ancestrais. Pesquisa FAPESP 196:1823.
International Union for Conservation of Nature and Natural Resources. 2015. The IUCN Red List of Threatened Species. < http://www.iucnredlist.org/>, 2015.

König H.E. \& Liebich H.G. 2004. Anatomia dos Animais Domésticos. $2^{\underline{a}}$ ed. Artmed Editora, Porto Alegre. 663p.

Leonel L.C.P.C., Lima T.C., Felipe R.L., Silva E.M., Silva G.A.O., Silva D.C.O., Carvalho-Barros R.A. \& Silva Z. 2013. Anatomia descritiva da traqueia do macaco-prego (Sapajus apella). Biotemas 26:179-183.

Lobo L.M., Santos A.C., Rosa R.A., Ambrosio C.E., Briani D.C., Costa G.M., Carvalho A.F. \& Mançanares C.A.F. 2014. Estudo macroscópico do aparelho digestório de Gracilinanus microtarsus (Wagner, 1842) (Mammalia: Didelphidae). Biotemas 27:109-120.

Lynch-Alfaro J.W., Mattews L., Bovette A.H., MacFarlan S.J., Hpilips K.A., Falótico T., Ottoni E., Verderane M., Izar P., Schulte M., Melin A., Fedigan L., Janson C. \& Alfaro M.E. 2012a. Anointing variation across wild capuchin populations: a review of material preferences, bout frequency and anointing sociality in Cebus and Sapajus. Am. J. Primatol. 74:299-314.

Lynch-Alfaro J.W., Boubli J.P., Olson L.E., Di Fiore A., Gutiérrez-Espeleta G.A., Chiou K.L., Schute M., Neitzel S., Ross V., Schwochow D., Nguyen M.T.T., Farias I., Janson C.H. \& Alfaro M.E. 2012b. Explosive Pleistocene range expansion leads to widespread Amazonian sympatry between robust and gracile capuchin monkeys. J. Biogeogr. 39:272-288.

Makungu M., Plessis W.M., Barrows M., Groenewald H.B. \& Koeppel K.N. 2014. Radiographic Thoracic Anatomy of the Ring-Tailed Lemur (Lemur catta). J. Med. Primatol. 43:144-152.

Oliveira M.M. \& Langguth A. 2006. Rediscovery of Marcgrave's capuchin monkey and designation of a neotype for Simia flavia Schreber, 1774 (Primates, Cebidae). Bolm Museu Nacional, Rio de Janeiro, 523:1-16.

Pais F.R.O. 2011. Avaliação odontológica em sincrânios de macaco-aranha (Ateles sp.). Dissertação de Mestrado em Ciência Animal, Centro Universitário Vila Velha, Vila Velha, ES. 141p.

Pinheiro L.L., Lima A.R., Muniz J.A., Imbeloni A., Fioreto E.T., Fontes R.F., Cabral R. \& Branco E. 2012. Anatomy and morphometric aspects of the trachea of Saimiri sciureus Linnaeus, 1758: knowledge for emergency procedures. Anais Acad. Bras. Ciências 84:973-977.

Pinto L.P. 2008. Ecologia alimentar do cuxiú-de-nariz-vermelho Chiropotes albinasus (Primates: Pitheciidae) na Floresta Nacional do Tapajós, Pará. Tese de Doutorado em Ecologia, Universidade Estadual de Campinas, Campinas, SP. 158p.

Pontes A.R.M., Malta A. \& Asfora P.H. 2006. A new species of capuchin monkey, genus Cebus erxleben (Cebidae, Primates): found at the very brink of extinction in the Pernambuco Endemism Centre. Zootaxa 1220:1-12.

Russell A.M., Rylands A.B. \& Wilson D.E. 2013. Handbook of the Mammals the World. Lynx, Barcelona. 953p.

Rylands A.B., Kierulff M.C. \& Russell A.B. 2005. Notes on the taxonomy and distributions of the tufted capuchin monkeys (Cebus, Cebidae) of South America. Lundiana 6:97-110.

Rylands, A.B. \& Kierulff, M.C.M. 2008. Cebus libidinosus (em Inglês). Lista Vermelha de Espécies Ameaçadas da IUCN de 2013. Versão 1. Página visitada em 12 de julho de 2013.

Rylands A.B. \& Kierulff M.C.M. 2013. Cebus libidinosus. Lista Vermelha de Espécies Ameaçadas da IUCN de 2013. Versão 1.

Silva Jr J.S. 2001. Especiação nos macacos-prego e caiararas, gênero Cebus, Erxbelen, 1777 (Primates, Cebidae). Tese de Doutorado, Universidade do Rio de Janeiro, RJ. 377p.

Souza A.C. 2013. Avaliação ultrassonográfica abdominal e descrição de Parâmetros normais em primatas não humanos. Dissertação de Mestrado em Ciências Veterinárias, Universidade Estadual de Santa Cruz, Ilhéus, BA. 104p.

Teixeira M.G., Ferreira A.F., Colaço A.A., Ferreira S.F., Benvenutti M.E.M. \& Queiroga F.L. 2013. Hematologic and blood chemistry values of healthy Cebus flavius kept in northeast of Brazil. J. Med. Primatol. 42:51-56.

Valença-Montenegro M.M. 2011. Ecologia de Cebus flavius (Shreber, 1774) em remanescentes de Mata Atlântica no estado da Paraíba. Tese de Doutorado em Ecologia Aplicada, Universidade de São Paulo, Piracicaba, SP. 133p. 